\title{
Pancreatic cancer diagnosed by the detection of gross hematuria due to urinary bladder metastasis: A case report
}

\author{
RISA KUBOTA $^{1}$, TOMOYASU TSUSHIMA ${ }^{1}$, KEISUKE DOI ${ }^{2}$, \\ YOUSUKE INOUE $^{3}$, YOKO SHINNO ${ }^{4}$ and TAKAHARU ICHIKAWA ${ }^{1}$
}

${ }^{1}$ Department of Urology, National Hospital Organization Okayama Medical Center, Okayama 701-1192; ${ }^{2}$ Department of Urology, Iwakuni Medical Center, Iwakuni, Yamaguchi 740-8510; ${ }^{3}$ Department of Urology, Hiroshima City Hospital, Hiroshima 730-8518; ${ }^{4}$ Department of Pathology, National Hospital Organization Okayama Medical Center, Okayama 701-1192, Japan

Received August 22, 2021; Accepted October 8, 2021

DOI: $10.3892 / \mathrm{mco} .2021 .2456$

\begin{abstract}
Pancreatic cancer is not easy to detect at its early stages due to difficulties in identifying symptoms at these stages. As it progresses, abdominal pain, loss of appetite, abdominal distension, jaundice and pain in the back, especially the lower back, might develop. Moreover, sudden onset or worsening of diabetes mellitus may be seen, which often prompts screening for the detection of pancreatic cancer. Since it rapidly spreads to surrounding tissues and organs, pancreatic cancer has a poor prognosis. However, metastasis to the bladder is rare, with few cases diagnosed on the basis of detecting gross hematuria. The current study presents a case of gross hematuria and exacerbated diabetes in a 90 -year-old woman. Cystoscopy revealed a non-papillary tumor in the posterior bladder wall. Pathological examination of bladder tumor specimens obtained via transurethral resection revealed adenocarcinoma. Subsequent systemic examinations revealed primary pancreatic cancer that had metastasized to the bladder. To the best of our knowledge, this is the second reported case of pancreatic cancer diagnosed based on the detection of gross hematuria due to bladder metastasis, since 1992.
\end{abstract}

\section{Introduction}

Currently, the number of patients with pancreatic cancer is gradually increasing both in Japan (https://ganjoho.jp/reg_stat/ statistics/data/dl/en.html) and in the United States (US) $(1,2)$. Pancreatic cancer accounts for $\sim 4 \%$ of all cancer cases in Japan, and was reported to be the fourth leading cause of

Correspondence to: Dr Risa Kubota, Department of Urology, National Hospital Organization Okayama Medical Center, 1711-1 Tamasu, Kita-ku, Okayama 701-1192, Japan

E-mail: m0r0s.0806.bye.gones@gmail.com

Key words: adenocarcinoma, bladder metastasis, diabetes mellitus, gross hematuria, pancreatic cancer, pathological examination, prognosis, transurethral resection cancer-related deaths in Japan in 2019 (1,3). In addition, the 5 -year survival rate for patients with pancreatic cancer in Japan is $\sim 8.5 \%$ (4). Similarly, in the US, pancreatic cancer is the third leading cause of cancer-related mortality, with a low 5 -year survival rate of 7-8\% (5). For most operable pancreatic tumors, surgery with curative intent is still the best treatment (6). However, the disease is usually in an advanced stage by the time of diagnosis involving metastasis to other organs. In such cases, chemotherapy or palliative therapy is a treatment option. Organs to which this cancer commonly spreads include the liver, lungs, stomach, bones and intestines (7). However, metastasis to the bladder is very rare. The current study presents a case of pancreatic cancer associated with gross hematuria due to bladder metastasis. If the chief complaint is hematuria, it may be important to perform a systemic search at an early stage, considering the possibility of metastatic bladder cancer.

\section{Case report}

Presentation and diagnosis. A 90-year-old woman presented with a 2-month history of gross hematuria associated with worsening of diabetes mellitus. The patient visited a local general physician and underwent an abdominal ultrasound in December 2015. However, no abnormal findings were found at that time. Approximately one week later, the patient was referred to Okayama Medical Center (Okayama, Japan) for the assessment of hematuria. Urine cytology showed increased chromatin and irregularly shaped epithelial cells in clusters, suggesting the possibility of malignancy. Cystoscopy revealed a $15-\mathrm{mm}$, non-papillary, broad-based tumor on the posterior wall of the bladder (Fig. 1). Complete blood cell count and other laboratory test results were within the normal ranges, except for blood sugar levels (209 mg/dl; normal range, 73-140 mg/dl) and hemoglobin A1c levels (9.2\%; normal range, 4.9-6.0\%).

A transurethral resection of the bladder tumor (TURBT) from the posterior wall was performed, and a histopathological diagnosis of invasive adenocarcinoma was established, as the main locus of the tumor was submucosal to intramuscular. The urothelium did not show any atypia. In addition, the adenocarcinoma appeared to grow from the muscular layer to 


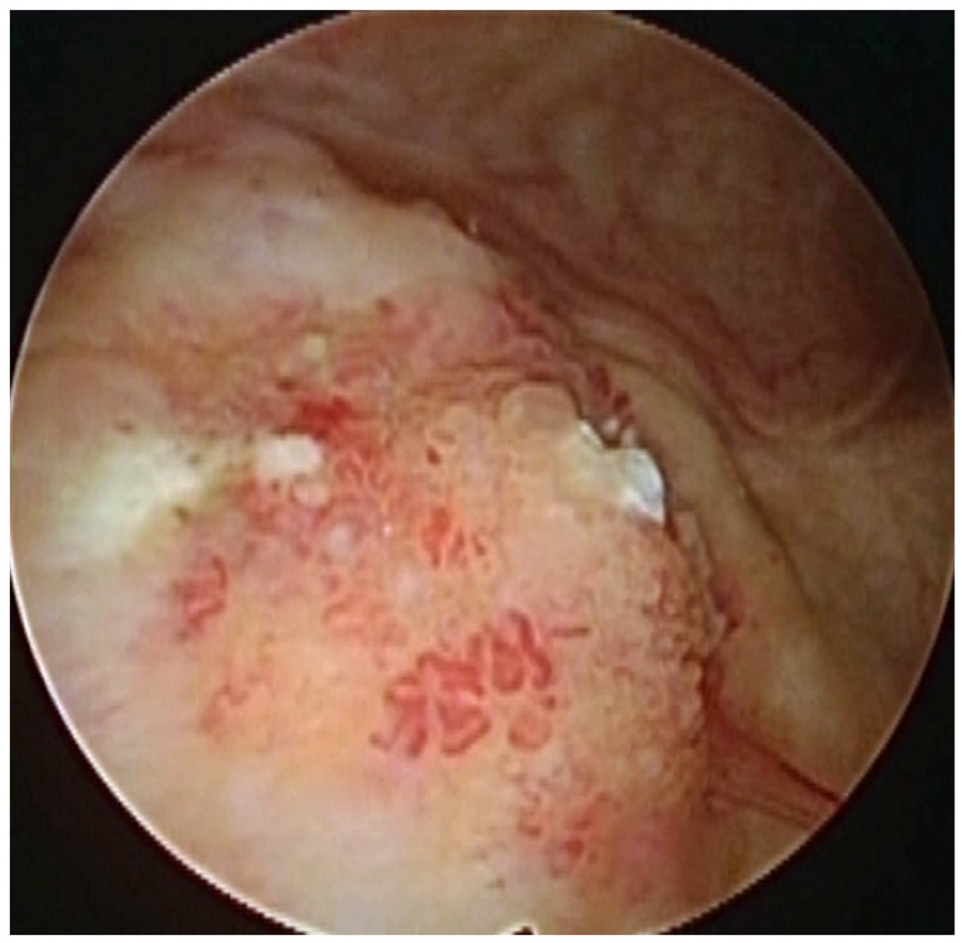

Figure 1. Cystoscopic findings. A 15-mm, non-papillary, broad-based tumor was located on the posterior wall of the bladder during cytoscopy.
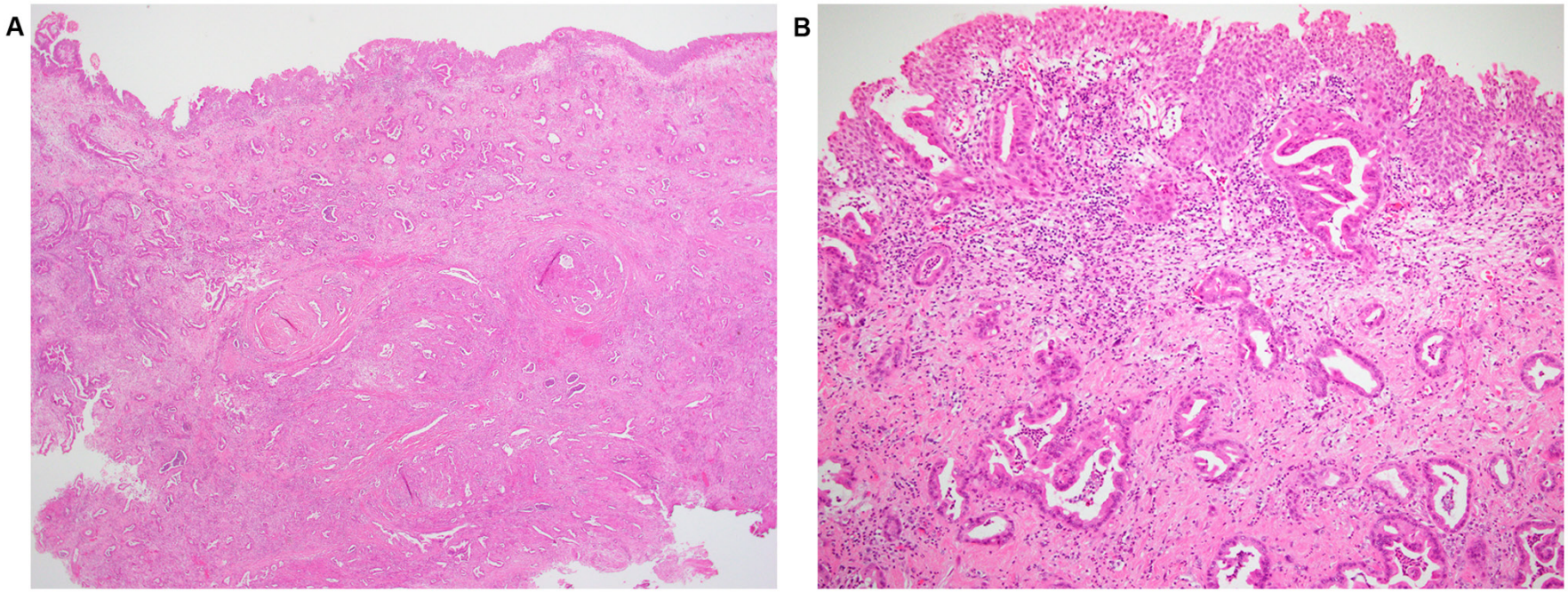

Figure 2. Pathological findings. Images of invasive adenocarcinoma showing the glandular pattern with cuboidal or columnar cells that are not continuous with the urothelial epithelium. Hematoxylin and eosin staining at (A) x20 and (B) x100 magnification.

the surface (Fig. 2). Therefore, it was necessary to determine whether this adenocarcinoma was primary or metastatic. Immunostaining for cytokeratin (CK)7 and S100p was positive. However, immunohistochemistry was negative for CK20, estrogen receptor, trans-acting T-cell-specific transcription factor GATA-3 and nuclear $\beta$-catenin. This staining pattern suggested that the lesion was unlikely to have a gynecological or a colorectal origin (Fig. 3). Contrast-enhanced computed tomography scan revealed a tumor with poor contrast effect in the pancreatic tail, which infiltrated the splenic vessels and metastasized to the left adrenal gland (Fig. 4). Based on these findings, a diagnosis of invasive ductal carcinoma of the pancreas, cT4N0M1, was established according to the Union for International Cancer Control (UICC) staging system (8), with metastasis to the urinary bladder and left adrenal gland. This carcinoma had not been revealed by ultrasound examination previously performed at the general clinic; it is possible that the doctor did not examine the pancreas region, as the chief complaint of the patient was hematuria. The patient declined aggressive treatment due to old age and chose to receive the best supportive care. The patient died 6 months after the diagnosis.

Tissue analysis. For analysis of the resected sample, the tissue was fixed with $10 \%$ neutral buffered formalin at room temperature overnight and paraffin embedded. Immunohistochemistry 

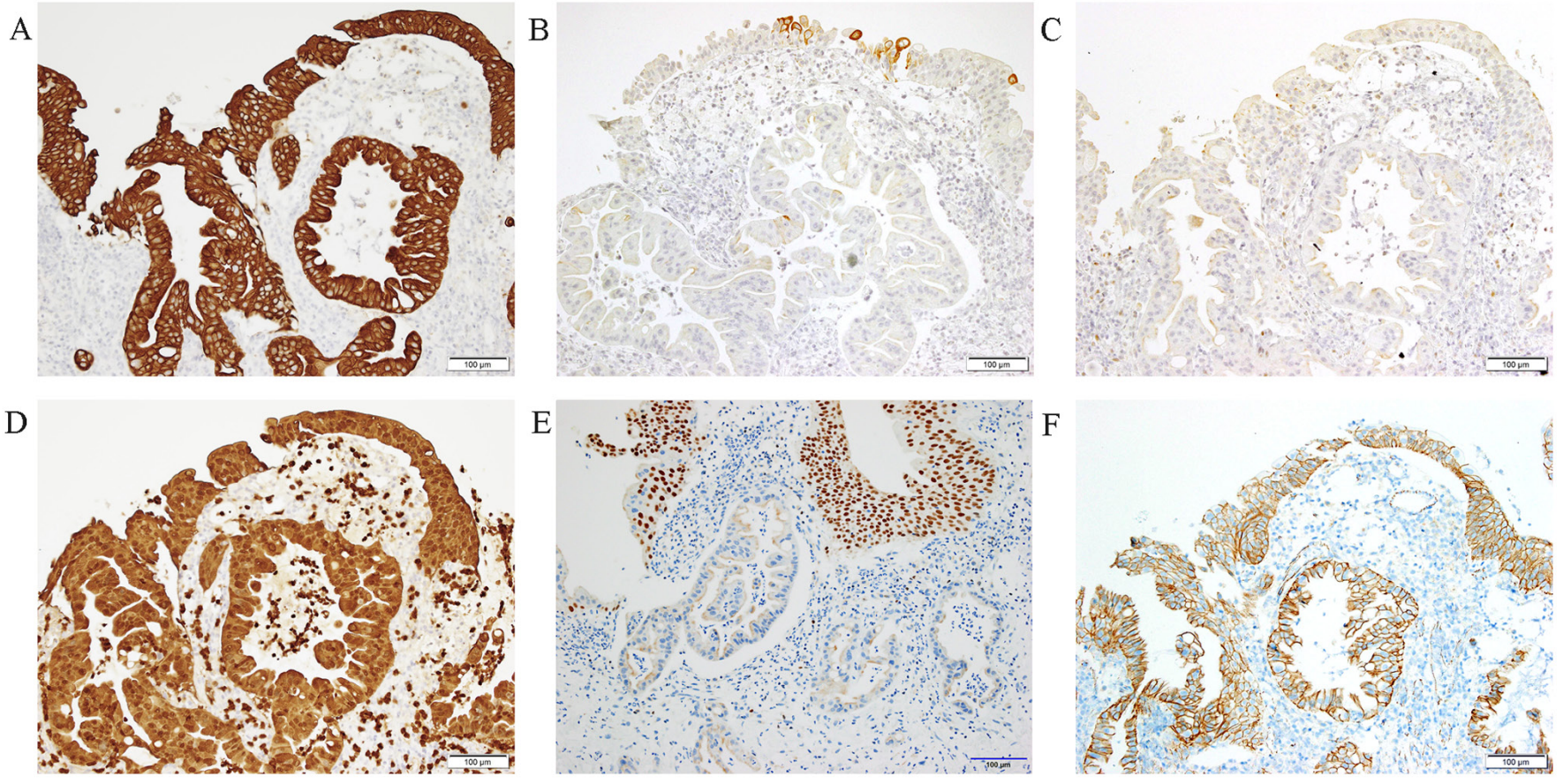

Figure 3. Immunostaining. (A) Positive for CK7, (B) negative for CK20, (C) negative for estrogen receptor and (D) positive for S100p. (E) Trans-acting T-cell-specific transcription factor GATA-3 was positive in the uroepithelium and negative in the adenocarcinoma component, and (F) $\beta$-catenin was positive in the membranes and negative in the nuclei. These staining patterns are compatible with invasive ductal carcinoma of the pancreas. CK, cytokeratin.
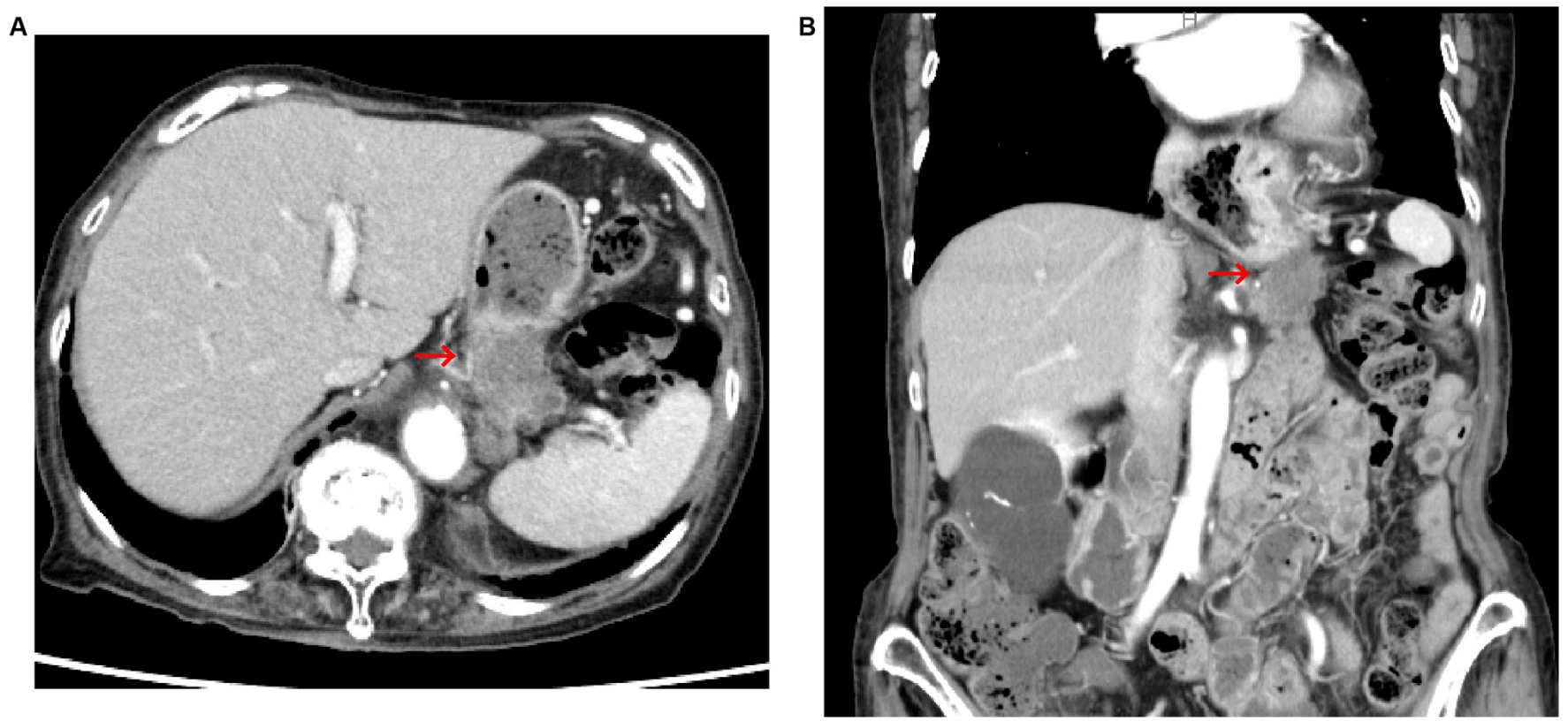

Figure 4. Contrast enhanced computed tomography. Arrows indicate a tumor with poor contrast effect in the pancreatic tail, infiltrating the splenic vessels and left adrenal gland mass. (A) Horizontal and (B) coronal sections.

was performed using $5-\mu$ m-thick sections on an automated immunohistochemistry system (Ventana BenchMark GX; Roche Diagnostics, Inc.) and the iVIEWDAB universal detection kit (Roche Diagnostics, Inc.). The primary antibodies were diluted with Antibody Diluent with Background Reducing Components (cat. no. S3022; Dako; Agilent Technologies, Inc.) containing carrier protein for blocking, accordingly the blocking time was the same as the incubation time, and the temperature was $35^{\circ} \mathrm{C}$. The antibody clone, supplier, dilution ratio, $\mathrm{CC} 1$ pretreatment time, incubation time and magnification set for each primary antibody were as follows: CK7 (OV-TL 12/30; Dako; Agilent Technologies, Inc.; 1:100; 60 min; 26 min; x200), CK20 (Ks 20.8; Dako; Agilent Technologies, Inc.; 1:100; $60 \mathrm{~min} ; 20 \mathrm{~min}$; x200), estrogen receptor (SP1; Ventana Medical Systems, Inc.; ready-to-use; $60 \mathrm{~min} ; 26 \mathrm{~min}$; x200), S100P (rabbit polyclonal; Atlas Antibodies AB; 1:3,000; $30 \mathrm{~min} ; 26 \mathrm{~min} ; \mathrm{x} 200$ ) and GATA-3 (L50-823; Biocare Medical LLC; 1:200; $60 \mathrm{~min} ; 32 \mathrm{~min}$; 
$\mathrm{x} 200$ ). The counterstaining was performed with hematoxylin for $5 \mathrm{~min}$ at room temperature. $\beta$-catenin ( $\beta$-catenin-1; Dako; Agilent Technologies, Inc.) was stained for at another facility, thus, the details of the staining conditions are unknown. The specimens were observed with a light microscope.

\section{Discussion}

The present study reports a rare case of pancreatic cancer associated with gross hematuria due to bladder metastasis. In Japan, the age-standardized incidence rate of pancreatic cancer in 2018 was 14.3 per 100,000 people, which was the sixth highest in the Japanese population (1). In 2019, the number of pancreatic cancer-related deaths was 18,124 among men and 18,232 among women, making it the fourth leading cause of death for men and the third for women (3). The risk factors for pancreatic cancer include family history, diabetes mellitus, chronic pancreatitis, intraductal papillary mucinous neoplasm and poor lifestyle choices, such as smoking (9).

Displacement of the left kidney and ureteral obstruction are common urological involvements in pancreatic cancer. However, metastasis to the bladder is rare. Warden et al (10) examined 20 cases of pancreatic cancer with urological involvement, excluding cases of bladder metastasis. Malignant tumors resulting in bladder metastasis are those of the pelvic organs, including urogenital, colon and rectal cancer. Of the malignancies that may metastasize to the bladder from distant organs, malignant melanoma, breast cancer and stomach cancer are common. However, metastasis from pancreatic cancer is very rare $(11,12)$, and has only been reported in 16 autopsy and clinical cases since 1927. Of these cases, 11 were detected in autopsies (11,13-17), and only five were diagnosed in living patients (7,18-21). Among the five clinical cases, gross hematuria due to bladder metastasis was observed in 3 individuals. Chiang et al (19) reported a case of pancreatic adenocarcinoma that metastasized to the bladder presenting with gross hematuria and jaundice. In the other two cases, metastasis to the bladder was observed during the treatment of pancreatic cancer $(20,21)$. To the best of our knowledge, after the study by Chiang et al in 1992 (19), this is the second case of bladder metastasis from pancreatic adenocarcinoma diagnosed by the detection of gross hematuria.

In conclusion, when an adenocarcinoma is diagnosed after TURBT, a differential diagnosis between primary bladder cancer and metastatic adenocarcinoma should be made. When gross hematuria is observed in patients with pancreatic cancer, invasion and metastasis to the urinary tract should be considered.

\section{Acknowledgements}

Not applicable.

\section{Funding}

No funding was received.

\section{Availability of data and materials}

The datasets used during the current study are available from the corresponding author on reasonable request.

\section{Authors' contributions}

TT and RK conceived and designed this case report. TI was in charge of the patient and decided their treatment. TT, KD and RK acquired data from the diagnostic imaging and histological results, and wrote the initial draft of the report. YI and RK acquired data from the diagnostic imaging and histological results, and all authors are also responsible for the analysis. YS performed additional immunostaining and contributed to the histological interpretation and discussion. TT revised the manuscript critically for important intellectual content. All authors read and approved the final version of the manuscript. RK and TT confirm the authenticity of all the raw data.

\section{Ethics approval and consent to participate}

As this is a case report and the substitute decision maker provided written informed consent for publication, it was granted exemption from approval by the Okayama Medical Center Clinical Research Review Committee (Okayama, Japan).

\section{Patient consent for publication}

As the patient had died, written informed consent was obtained from the family for the publication of the present report.

\section{Competing interests}

The authors declare that they have no competing interests.

\section{References}

1. Cancer Information Service, National Cancer Center, Japan: National Cancer Registry (Ministry of Health, Labour and Welfare). https://ganjoho.jp/reg_stat/statistics/data/dl/en.html. Accessed September 29, 2021.

2. American Cancer Society: Cancer Facts and Figures 2021. American Cancer Society, Atlanta, GA, 2021

3. Cancer statistics Cancer Information Service, National Cancer Center, Japan (Vital Statistics of Japan, Ministry of Health, Labour and Welfare). https://ganjoho.jp/reg_stat/statistics/data/ dl/en.html. Accessed September 29, 2021.

4. Monitoring of Cancer Incidence in Japan - Survival 2009-2011 Report (Center for Cancer Control and Information Services, National Cancer Center, 2020). https://ganjoho.jp/reg_stat/ statistics/data/dl/en.html. Accessed September 29, 2021.

5. Siegel RL, Miller KD and Jemal A: Cancer Statistics, 2017. CA Cancer J Clin 67: 7-30, 2017.

6. Tempero MA, Malafa MP, Al-Hawary M, Asbun H, Bain A, Behrman SW, Benson AB III, Binder E, Cardin DB, Cha C, et al: Pancreatic adenocarcinoma, Version 2.2017, NCCN clinical practice guidelines in oncology. J Natl Compr Canc Netw 15: 1028-1061, 2017.

7. Shah A, Korrapati P, Siegel J and Kasmin F: Rare metastasis of primary pancreatic adenocarcinoma to the bladder. ACG Case Rep J 5: e27, 2018.

8. Amin MB, Edge SB, Greene FL, Byrd DR, Brookland RK, Washington MK, Gershenwald JE, Compton CC, Hess KR, Sullivan DC, et al American Joint Committee on Cancer: AJCC Cancer Staging Manual. 8th edition. Springer, New York, NY, 2017. https://doi.org/10.1007/978-3-319-40618-3.

9. Yamaguchi K, Okusaka T, Shimizu K, Furuse J, Ito Y, Hanada K, Shimosegawa T, Yamaguchi K, Okusaka T, Shimizu K, et al; Committee for revision of clinical guidelines for pancreatic cancer of Japan Pancreas Society: EBM-based Clinical Guidelines for Pancreatic Cancer (2013) issued by the Japan Pancreas Society: A synopsis. Jpn J Clin Oncol 44: 883-888, 2014. 
10. Warden SS, Fiveash JG Jr, Tynes WV II and Schellhammer PF II Urologic aspects of pancreatic adenocarcinoma. J Urol 125: 265-267, 1981.

11. Goldstein AG: Metastatic carcinoma to the bladder. J Urol 98: 209-215, 1967.

12. Velcheti V and Govindan R: Metastatic cancer involving bladder: A review. Can J Urol 14: 3443-3448, 2007.

13. Klinger ME: Secondary tumors of the genito-urinary tract. J Urol 65: 144-153, 1951.

14. Kiefer ED: Carcinoma of the pancreas. Arch Intern Med 40 $1-29,1927$.

15. Sommers SC and Meissner WA: Unusual carcinomas of the pancreas. AMA Arch Pathol 58: 101-111, 1954.

16. Bell ET: Carcinoma of the pancreas. I. A clinical and pathologic study of 609 necropsied cases. II. The relation of carcinoma of the pancreas to diabetes mellitus. Am J Pathol 33: 499-523, 1957.

17. Sheehan EE, Greenberg SD and Scott R Jr: Metastatic neoplasms of the bladder. J Urol 90: 281-284, 1963.
18. van Dyk D, Lang R, Jutrin Y, Shapira J and Ravid M: Bizarre urologic manifestations of pancreas carcinoma. Hepatogastroenterology 27: 62-63, 1980.

19. Chiang KS, Lamki $\mathrm{N}$ and Athey PA: Metastasis to the bladder from pancreatic adenocarcinoma presenting with hematuria. Urol Radiol 13: 187-189, 1992.

20. Lavelle RS, Williams SB and O'Leary MP: An 84-year-old female with gross hematuria. Urology 77: 533-534, 2011.

21. Cellini M and Deighton DA: Radiological case: Non-papillary bladder metastasis from pancreatic adenocarcinoma. Appl Radiol 43: 74-76, 2014.

cC)(7) $\fallingdotseq$ This work is licensed under a Creative Commons cc) Attribution-NonCommercial-NoDerivatives 4.0 International (CC BY-NC-ND 4.0) License. 